\title{
Development of the fabrication process of carbon nanotube reinforced polylactide (PLA) nanofiber and evaluation of its mechanical properties
}

\author{
K. Tanaka, M. Hashimoto, M. Nagura \& T. Katayama \\ Department of Biomedical Engineering, Doshisha University, Japan
}

\begin{abstract}
Due to the high surface area to volume ratio, nanofibers are expected to be used for a wide range of applications such as tissue engineering, filter media and reinforcements in composites. The electrospinning technique is a simple method for the fabrication of nanofibers. In this technique, a polymer solution is blown out from a needle hole, while an electric field is applied between a polymer solution and a target. During the electrospinning process, the solvent evaporates and polymer nanofibers are fabricated on the target.

To add special functions to nanofibers, functional particle such as calcium carbonate and $\mathrm{TiO}_{2}$ were reported to be used as nano-fillers for nanofibers. Carbon nanotube (CNT), which has excellent mechanical, electrical, chemical resistance and electromagnetic properties, was reported to be reinforcement for bulk polymer resin and CNT is anticipated to be a reinforcing nano-fillers. However, due to challenges such as manipulating extremely small fibers, preparing samples of single-nanofiber and sourcing for accurate and sensitive force transducer, it is difficult to evaluate the mechanical properties of CNT reinforced nanofibers.

In this study, CNT/PLA nanofibers were fabricated by the electrospinning method using the electrical potentials difference between two $\mathrm{Cu}$ strips of target and the mechanical properties of CNT/PLA nanofiber were evaluated. The morphology of the composite fibers and the dispersion of the CNT within the fibers were observed using transmission electron microscopy (TEM). The tensile test of PLA and CNT/PLA single nanofiber were successfully conducted using a nano-scale tensile testing machine. The tensile strength of PLA and CNT
\end{abstract}


(0.5 wt.\%)/PLA nanofiber were $95 \pm 23 \mathrm{MPa}$ and $102 \pm 35 \mathrm{MPa}$, respectively. The addition of CNT for $0.5 \mathrm{wt} . \%$ to PLA did not affect the mechanical property of PLA nanofiber.

Keywords: electrospinning, nanofiber, poly lactic acid (PLA), carbon nanotube (CNT), tensile test.

\section{Introduction}

Polymer nanofibers, which have small fiber diameters compared with microfibers having fiber diameters of micrometers, are expected to be used for a wide range of applications such as tissue engineering [1] and filter media [2], and so on, since polymer nanofibers have such characteristics as nanosize effects, high surface area to volume ratio, and supramolecular arrangement effects [3]. The electrospinning is a common method for the fabrication of nanofibers. In this process, when high-voltage is applied between polymer solution and the target, polymer solution was blown out from a needle hole. During the electrospinning process, the solvent evaporates and polymer nanofibers are fabricated on the target [3-5]. Many thermoplastic polymers such as polyacrylonitrile [6], polyimide [7], and polyvinyl alcohol [8] have been already succeeded in nanofibers formation by electrospinning method. When they are used for scaffolds, PLA, which is biocompatible and has high strength, has been attracted attentions. In addition, to add special functions to nanofibers, functional particles such as calcium carbonate [9], oxidized titanium [10], and carbon nanotube (CNT) [11] were reported to be used as nano-fillers. Among these nanofillers, CNT, which has excellent mechanical, electrical, thermal, and electromagnetic properties, has been reported to be reinforcement for bulk polymer resin and is also anticipated to be a reinforcing nano-fillers [12]. While, due to some challenges such as high cohesive property of CNT, manipulating extremely small fibers, preparing specimens of single-nanofiber, and sourcing for accurate and sensitive force transducer, it is difficult to evaluate mechanical properties of CNT reinforced nanofibers [13].

In this study, fabrication methods of nanofibers with dispersed CNT were examined, and PLA and carbon nanotube reinforced polylactide (CNT/PLA) single nanofibers were fabricated by electrospinning method that used the electrical potential differences between two $\mathrm{Cu}$ strips of the target, then the mechanical properties of PLA and CNT/PLA nanofibers were clarified.

\section{Materials and experimental procedures}

\subsection{Materials and fabrication process}

PLA (weight-average molecular weight, $\mathrm{Mw}=2.7 \times 10^{5} \mathrm{~g} / \mathrm{mol}$ ) was used in this study. Multiwalled carbon nano-tubes (MWCNT, Showa Denko) with $6 \mu \mathrm{m}$ in length, $150 \mathrm{~nm}$ in average diameter and 10-500 in aspect ratio were used as nanofillers. Dichloromethane (DCM) was used as solvent and dimethylformamide (DMF) was used to control solution conductivity. 
The polymer solution was prepared by adding PLA pellets (8 wt.\%) to DCM/DMF (8:2 weigh/weigh: w/w), then the solvent was stirred at $30^{\circ} \mathrm{C}$ for $45 \mathrm{~min}$. In the case for carbon nanotube reinforced polylactide (CNT/PLA), CNT $(0.5$ wt. $\%)$ was added to the solvent, two kinds of stirring methods were used in order to investigate difference of CNT dispersion.

\section{Stirring condition (A)}

PLA pellet was added to DCM/DMF $(8: 2 w / w)$ solvent which was stirred at $30{ }^{\circ} \mathrm{C}$ for $45 \mathrm{~min}$. Then, CNT was added to the solution and stirred for $20 \mathrm{~min}$. Stirring condition (B)

CNT was added to DMF and stirred at room temperature for 12 hours. Then, PLA pellet was added to DCM and stirred at $30^{\circ} \mathrm{C}$ for $45 \mathrm{~min}$. Then, two solutions were mixed and stirred at room temperature for 12 hours.

The PLA and CNT/PLA solutions were spun into nanofibers by using an electrospinning system (NEU, Kato Tech). Fig. 1 shows schematic diagram of the electrospinning process. Electrospinning was conducted at room temperature $\left(20 \pm 3{ }^{\circ} \mathrm{C}\right)$ and at $35-40 \%$ relative humidity. Potential differences between the needle and the target were set at 10,16 and $24 \mathrm{kV}$ and feed speed of the syringe was set at $3.0 \times 10^{-4} \mathrm{ml} / \mathrm{sec}$. PLA and CNT/PLA nonwoven nanofibers were spun on the target of aluminum foil, which was used for the observation of the structure and morphology of PLA and CNT/PLA nanofibers. To fabricate aligned single nanofibers for the tensile test, two $\mathrm{Cu}$ strips, which were placed parallel with $30 \mathrm{~mm}$ distance, were used for the target (as shown in Fig. 2). The potential difference between two $\mathrm{Cu}$ strips was set at $3 \mathrm{~V}$ [14].

A scanning electron microscopy (SEM, JSM-6390LT, JEOL), was used to observe the structure and morphology of PLA and CNT/PLA nanofibers that were spun by the electrospinning system. Specimens were covered with platinum by sputter-coating and observed at an accelerating voltage of $15 \mathrm{kV}$. A transmission electron microscopy (TEM, JEM2100F, JEOL) was used to observe the dispersion of CNT in the fibers at an accelerating voltage of $200 \mathrm{kV}$. A differential scanning calorimeter (DSC, DSC-60, SHIMADZU) was used to measure the crystallinity and melting behavior of the fiber specimens.
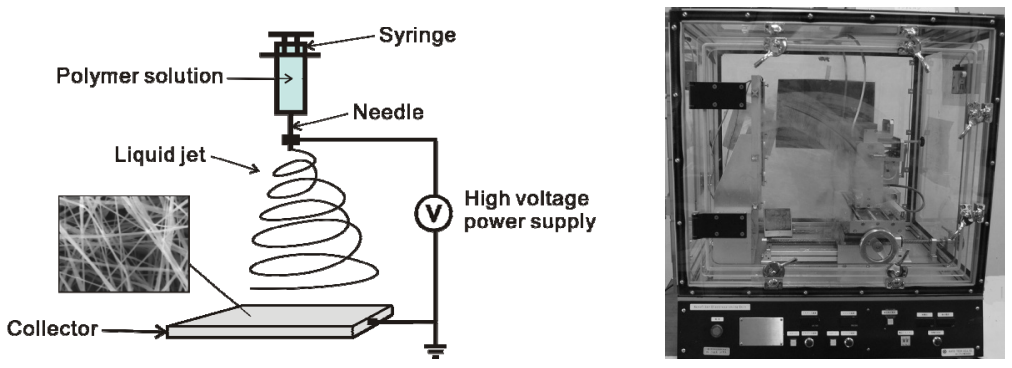

Figure 1: Schematic drawing of the electrospinning process and electrospinning unit. 

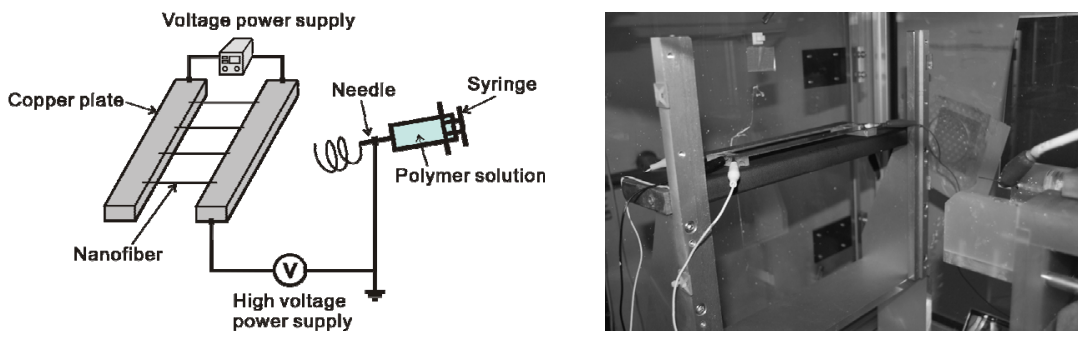

Figure 2: Device for the fabrication of aligned single nanofiber.

\subsection{Mechanical properties of single nanofibers}

The aligned single nanofibers were used for specimens for the single fiber tensile tests. A single nanofiber was glued to a tab with conductive tapes as shown in Fig. 3. The tensile tests of nanofibers were conducted by using a nano tensile testing machine (NanoTensile, Hysitron), at a displacement rate of $10 \mu \mathrm{m} / \mathrm{s}$ $(0.6 \mathrm{~mm} / \mathrm{min})$. This testing machine has $500 \mathrm{mN}$ load range and $3.5 \mu \mathrm{m}$ noise floor. After the tensile tests, the diameter of the nanofibers, which were bonded to conductive tape, was measured by using the SEM. The average value of diameters, which were measured at five points, was used to calculate the tensile strength.

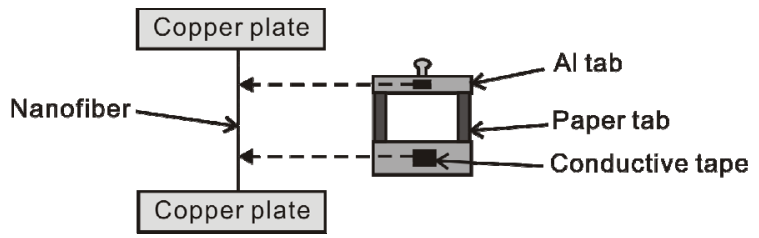

Figure 3: Procedure of specimen preparation.

\section{Results and discussions}

\subsection{Morphology of nanofibers}

Fig. 4 shows the observation of polymer solution that was observed by an optical microscope. Significant differences in the dispersion of CNT in polymer solution by the differences of the stirring methods were observed. Stirring method (B), which is longer in stirring time of DMF and CNT than stirring method (A), was confirmed more clearly than stirring method (A) that masses of CNT were not remained and dispersed in polymer solution. 


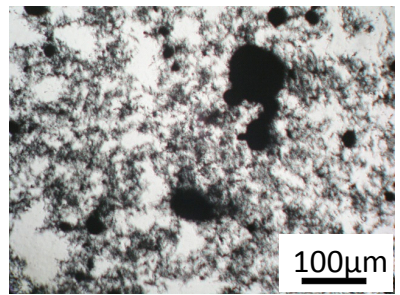

(1) Stirring condition (A)

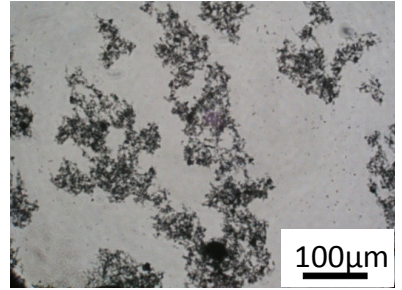

(2) Stirring condition (B)

Figure 4: Optical microscope images.

Fig. 5 shows the TEM observations of CNT/PLA nanofibers which were stirred by stirring method (A) and (B). CNT was contained in PLA nanofiber. In addition, differences in the orientation of CNT in nanofibers due to the differences of the stirring methods were observed. CNT was not oriented axially relative to PLA and mass of CNT was observed in the nanofibers for stirring method (A). CNT was oriented axially relative to PLA for stirring method (B).
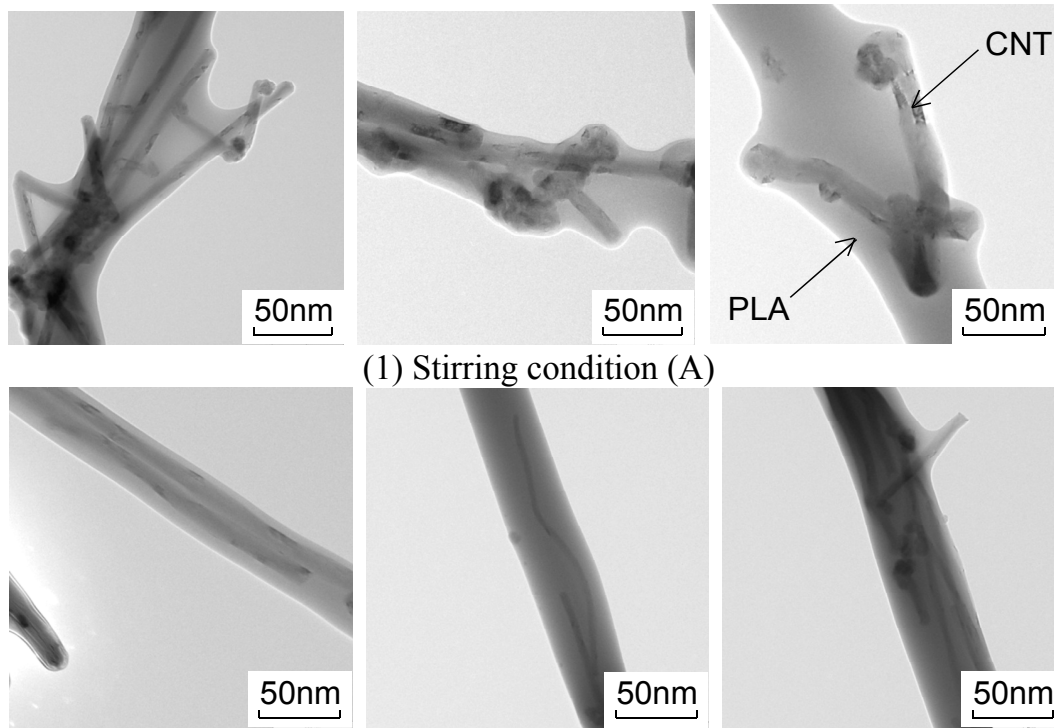

(1) Stirring condition (A)

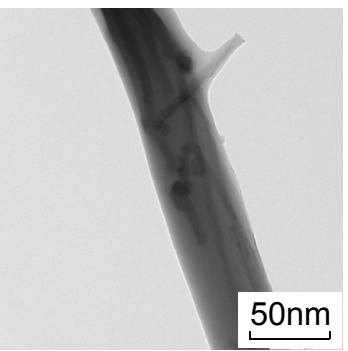

(2) Stirring condition (B)

Figure 5: TEM images of nanofibers.

Figs 6 and 7 show the SEM observations of PLA and CNT/PLA nonwoven nanofibers with 10,16 , and $24 \mathrm{kV}$ of applied voltages. PLA nanofibers with relatively-uniform diameters were fabricated at $16 \mathrm{kV}$ of applied voltage in PLA and at $10 \mathrm{kV}$ applied voltage in CNT/PLA nanofibers. For both PLA and CNT/PLA nanofibers, the higher voltages were applied, the more the diameters 
of the fibers were varied. A cause that applied voltages to fabricate uniform nanofibers was different between PLA and CNT/PLA, was considered to be the differences of the electrical conductivity of polymer solution. As CNT has good electrical conductivity, it is considered that the electric conductivity of polymer solution was increased by adding CNT, and then differences in the fabrication conditions were observed.

DSC was used to measure the crystallinity and melting behavior of the fiber specimens. The heating curves are presented in Fig. 8. The glass transition

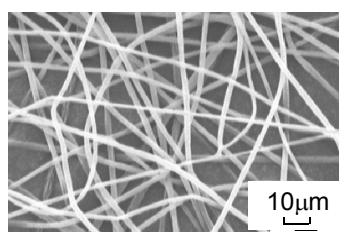

(a) $10 \mathrm{kV}$

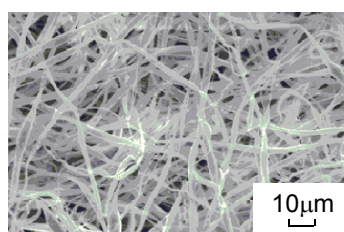

(b) $16 \mathrm{kV}$

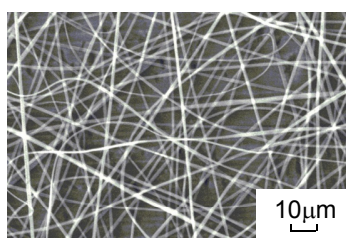

(c) $24 \mathrm{kV}$

Figure 6: $\quad$ SEM images of PLA nonwoven nanofibers.

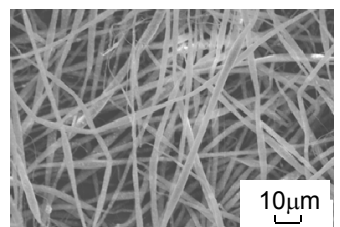

(a) $10 \mathrm{kV}$

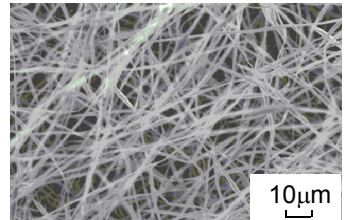

(b) $16 \mathrm{kV}$

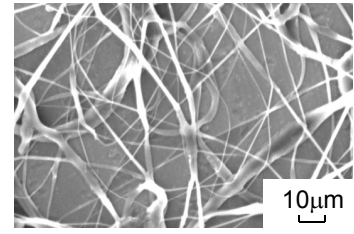

(c) $24 \mathrm{kV}$

Figure 7: SEM images of CNT/PLA nonwoven nanofibers.

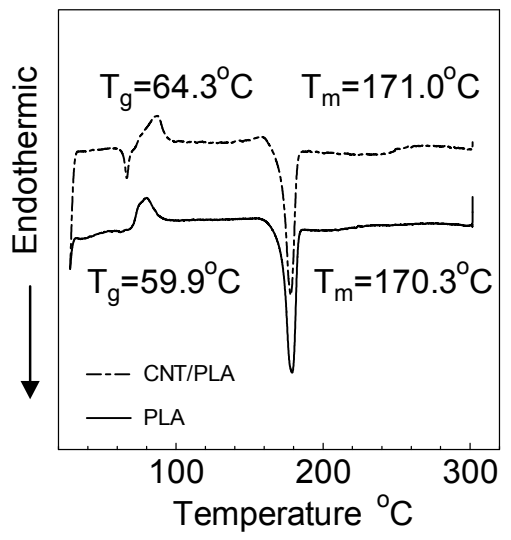

Figure 8: Heating curves for PLA and CNT/PLA nanofiber. 
temperatures, melting points and crystallinity values of PLA nanofibers were recorded at $59.9^{\circ} \mathrm{C}, 170.3^{\circ} \mathrm{C}$ and $45.5 \%$ respectively. The glass transition temperatures, melting points and crystallinity values of CNT/PLA nanofibers were recorded at $64.3^{\circ} \mathrm{C}, 171.0^{\circ} \mathrm{C}$ and $41.7 \%$ respectively. Glass transition temperature of CNT/PLA nanofibers was higher than that of PLA nanofiber and crystallinity values of CNT/PLA nanofibers were lower than those of PLA nanofiber. On the other hand, melting points of PLA nanofibers were not affected by the addition of CNT.

\subsection{Mechanical properties of single nanofibers}

Fig. 9 shows the result of tensile tests. The tensile strengths of PLA and CNT/PLA nanofibers were $95 \pm 23 \mathrm{MPa}$ and $102 \pm 35 \mathrm{MPa}$ respectively. The addition of CNT by $0.5 \mathrm{wt} \%$ to PLA did not affect the mechanical properties of PLA.

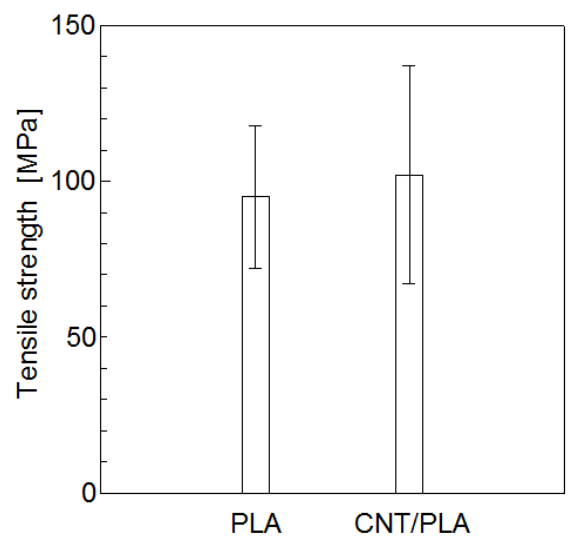

Figure 9: Tensile strength in PLA and CNT/PLA.

\section{Conclusion}

Fabrication methods of nanofibers with dispersed CNT were examined and PLA and CNT/PLA single nanofibers were fabricated by electrospinning method that used the electrical potential differences between two $\mathrm{Cu}$ strips of the target, then the mechanical properties of PLA and CNT/PLA nanofibers were clarified. The investigations yielded the following conclusions:

(1) The tensile strengths of PLA and CNT/PLA nanofibers were $95 \pm 23 \mathrm{MPa}$ and $102 \pm 35 \mathrm{MPa}$ respectively. The addition of CNT by $0.5 \mathrm{wt} \%$ to PLA did not affect the mechanical properties of PLA nanofibers.

(2) By adding CNT, glass transition temperature of PLA nanofibers was higher than that of CNT/PLA nanofiber and crystallinity values of PLA 
nanofibers were lower than those of CNT/PLA nanofibers. On the other hand, melting point of PLA nanofibers was not affected by the addition of CNT.

\section{Acknowledgements}

This work was partially supported by a research project on "Research and Development Center for Advanced Composite Materials" of Doshisha University and MEXT (the Ministry of Education, Culture, Sports, Science and Technology, Japan) - Supported Program for the Strategic Research Foundation at Private Universities, 2013-2017, the project S1311036.

\section{References}

[1] F. Andrzej et al., Mapping critical sites in collagen II for rational design of gene-engineered proteins for cell-supporting materials, Journal of Biomedical Materials Research 57(1), 48-58, 2001.

[2] K. Graham et al., Polymeric Nanofibers in Air Filtration Applications Fifteenth Annual Technical Conference \& Expo of the American Filtration \& Separations Society, Galveston, 2002.

[3] Z. M. Huanga, A review on polymer nanofibers by electrospinning and their applications in nanocomposites, Composites Science and Technology 63, 2223-2253, 2003.

[4] Y Ishii et al., A new electrospinning method to control the number and a diameter of uniaxially aligned polymer fibers, Materials Letter, 62, 33703372, 2008.

[5] S. Ramakrishna et al., An Introduction to Electrospinning and Nanofibers, World Scientific Publishing Co. Pte. Ltd, 1-21, 2005.

[6] S. Y. Gu et al., Process optimization and empirical modelling for electrospun polyacrylonitrile (PAN) nanofiber precursor of carbon nanofibers, European Polymer Journal 41, 2559-2568,2005.

[7] D. Chen et al., Electrospinning Fabrication of High Strength and Toughness Polyimide Nanofiber Membranes Containing Multiwalled Carbon Nanotubes, J. Phys. Chem. B, 113 (29), 9741-9748, 2009.

[8] B. Ding et al., Preparation and Characterization of a nanoscale poly(vinyl alcohol) fiber aggregate produced by an electrospinning method, 40 (13), 1261-1268, 2002.

[9] K. Fujihara et al., Guided bone regeneration membrane made of polycaprolactone/calcium carbonate composite nano-fibers, Biomaterials, 26, 4139-4147, 2005.

[10] H. R. Panta et al., Electrospun nylon-6 spider-net like nanofiber mat containing $\mathrm{TiO} 2$ nanoparticles, a multifunctional nanocomposite textile material, Journal of Hazardous Materials, 185(1), 124-130, 2011.

[11] S. Shao et al., Osteoblast function on electrically conductive electrospun PLA/MWCNTs nanofibers, Biomaterials, 32, 2821-2833, 2011. 
[12] K. T. Lau et al., The revolutionary of new advanced materials - carbon nanotube composite, Composite, Part B 33, 263-277, 2002.

[13] E. P. S. Tan et al., Mechanical characterization of nanofibers - A review, Composites Science and Technology 66, 1102-1111, 2006.

[14] K. Tanaka et al., Development of electrospinning method for tensile test of single nanofibers, WIT Transactions on The Built Environment, 112, 117$185,2010$. 